\title{
Editorial
}

\section{Myocardial supply and demand}

The dependence of myocardial ischaemia on the balance between supply and demand (that is, adequacy of blood flow for a certain rate of cardiac work) is a well established concept. However, the basic mechanisms of autoregulation and metabolic regulation on which the concept depends may be less familiar. Other measures such as coronary flow reserve and potency of coronary vasodilators also depend on the coronary flow control diagram illustrated in fig 1 .

It is obvious that coronary arterial occlusion impairs supply but this is certainly not so obvious if there is coronary artery stenosis, because of the presence of autoregulation. Autoregulation is the relation between coronary arterial blood flow and perfusion pressure indicated by the sigmoid curves in fig 1 . Over a wide normal range of pressures, flow changes very little. When the demand is increased-for example, by increased heart rate, these curves move upwards in parallel from 1 to 2 to 3 , etc. If the coronary vascular bed is fully dilated-for example, by adenosine, the flow-pressure curve goes to the maximum straight line relation.

\section{Effect of increased demand on coronary flow reserve}

Coronary flow reserve is determined by measuring the increase in flow that occurs when maximum vasodilatation is induced. If one starts from point $A$ in fig 1 , the reserve is given by the length of the arrow AC. If, however, the metabolic rate in the control situation is high-for exam-

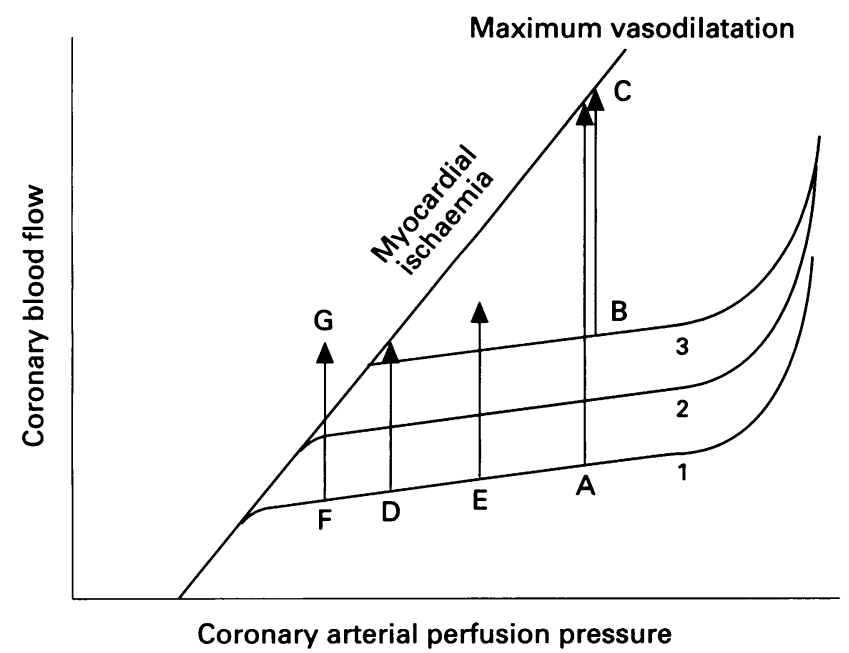

Figure 1 The sigmoid curves 1, 2, and 3 show how, for three different levels of demand, the supply (coronary blood flow) depends on coronary perfusion pressure. Over a wide normal range of perfusion pressure, flow changes little because of autoregulation of flow. An increase in demand chanses a parallel upward shift of the autoregulation curve from 1 to 2 to 3 , etc. The straight line indicates the maximum flow of which the coronary. vascular bed is capable for different perfusion pressures. If demand requires flows above this line, ischaemia develops. ple, at point $B$, the coronary flow reserve is given by the length of the arrow BC.

Thus coronary flow reserve is reduced; however, this has nothing to do with any incapacity of either the epicardial coronary arteries or the microcirculation.

\section{Effect of coronary artery stenosis on coronary flow reserve}

A stenosis in an epicardial artery will have no effect unless it produces a pressure gradient. Now that pressure transducers on coronary angioplasty (PTCA) guidewires are available, it is important wherever possible to measure the downstream pressure and to pay attention exclusively to stenoses where there is a pressure drop across the lesion. In fig 1 , the distal pressure could be at point $\mathrm{D}$. The coronary flow reserve is given by the length of the arrow from $\mathrm{D}$ - that is, reduced compared with AC because of an important epicardial coronary artery stenosis.

\section{Effect of microvascular dysfunction on coronary flow reserve}

A result such as that represented by the arrow from point $E$ in fig 1 , which fails to reach the expected line of maximum dilatation, implies a problem distal to the epicardial arteries. This indicates either atheromatous disease of small arteries not accessible to a pressure transducer, or a true microvascular defect as in microvascular angina.

\section{The maximum vasodilatation line is the ischaemia line}

Consider a critical coronary arterial stenosis that gives a distal pressure at point $F$ and increase the demand to beyond level 3. The required flow is at point $G$ but the heart cannot achieve it because it is beyond the maximum vasodilatation line. Only now is supply inadequate for demand so that ischaemia develops. Any target value above and to the left of the maximum vasodilatation line will produce ischaemia. It follows that the higher the distal pressure, the more the increase in demand required to produce ischaemia, and conversely, the higher the pressure gradient across the coronary stenosis (the lower the distal pressure), the less the demand increase needed to induce ischaemia and the worse is effort induced angina.

\section{Metabolic regulation}

For a given perfusion pressure-for example, that at point $A$ in fig 1 , metabolic regulation is defined by the coronary blood flow as a function of myocardial oxygen consumption as illustrated by the continuous line in fig 2 . This line is obtained by plotting the flows obtained on increasing demand along the points $\mathrm{A}, \mathrm{B}, \mathrm{C}$ up to the maximum vasodilatation line in fig 1 . This is the "supply-demand 


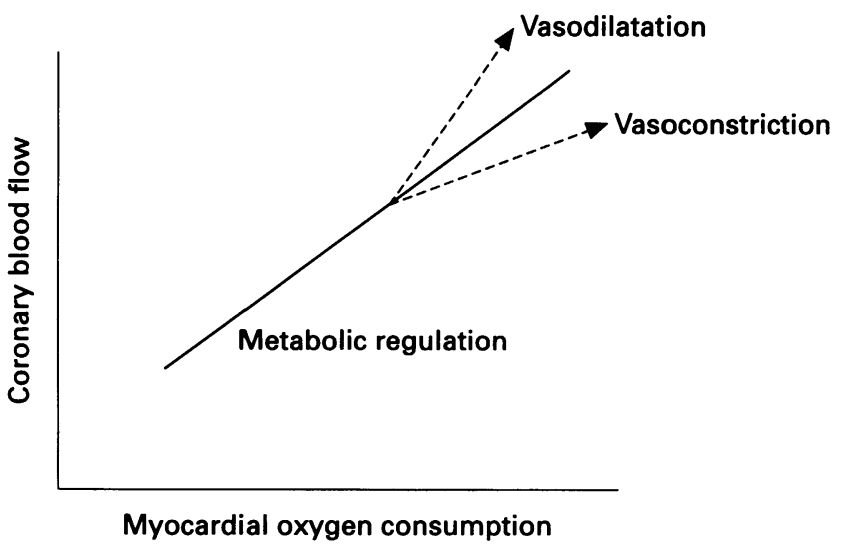

Figure 2 The supply-demand diagram. Coronary flow (supply) is a linear function of myocardial oxygen consumption (demand) for a given perfusion pressure. Vasoconstriction deflects the relation downward, vasodilatation deflects it upward. (Adapted from reference 1).

diagram" showing coronary blood flow (supply) as a function of myocardial oxygen consumption (demand).

A notable contribution was made to understanding the effects on the coronary circulation other than autoregulation and metabolic regulation by Mohrman and Feigl. ${ }^{1}$ Having defined the metabolic regulation line given by the continuous line in fig 2 , they showed that sympathetic stimulation or noradrenaline (from a starting point on the line) gave a result illustrated by the dashed arrow below the line. The point to note is that, although the coronary blood flow increases, this is a vasoconstriction-it is not possible to interpret a change of coronary blood flow on its own, correction must be made for any changes in myocardial oxygen consumption that accompany the intervention. In this particular case, sympathetic stimulation accelerated heart rate and cardiac metabolic rate to increase blood flow but not as much as would have occurred in the absence of vasoconstriction.
The supply-demand diagram in the assessment of coronary vasodilators

If, instead of following the vasoconstriction arrow in fig 2 , an intervention simply moved the heart up the continuous line, the increase in coronary blood flow might erroneously be interpreted as vasodilatation. However, only metabolic vasodilatation has occurred and the intervention is completely neutral from the point of view of coronary vessel tone. True vasodilatation is indicated by the dashed arrow above the solid line in fig 2 .

Vergroesen and colleagues in this issue ${ }^{2}$ present a refinement of this method of analysis as a method for true assessment of coronary vasodilating drugs. Essentially, they have simplified fig 2 by plotting everything from the midpoint on the metabolic regulation line as change in coronary blood flow versus change in myocardial oxygen consumption, with the metabolic change determined by pacing in patients. The slope of this line is the supply-demand ratio. The effect of the putative coronary vasodilator is then compared and true vasodilatation indicated by a shift away from the metabolic vasodilatation line.

I strongly commend this approach to cardiologists for the assessment of coronary vasodilator drugs. However, caution should be exercised in patients with critical stenoses of epicardial arteries with a measurable pressure drop across the lesion, because any intervention will change the distal pressure-that is, the perfusion pressure of the coronary vascular bed on the abscissa of fig 1 . Such patients should be excluded from this type of investigation.

MARK I M NOBLE

National Heart and Lung Institute,

Imperial College School of Medicine at Charing Cross Hospital, Fulham Palace Road,

London W6 8RF, UK

1 Mohrman DE, Feigl EO. Competition between sympathetic vasoconstriction and metabolic vasodilation in the canine coronary circulation. Circulation 1978;42:79-86

2 Vergroesen I, Kal JE, Spaan JAE, Van Wezel HB. Myocardial oxygen supply demand ratio as reference for coronary vasodilatory drug effects in humans. Heart 1997;78:117-21. 